Volume 70, Number 1, Pages 143-155(2021)

DOI: $10.31801 /$ cfsuasmas.726225

ISSN 1303-5991 E-ISSN 2618-6470

Received by the editors: April 24, 2020; Accepted: November 20, 2020

\title{
SOME PROPERTIES OF A NEW CLASS OF ANALYTIC FUNCTIONS DEFINED VIA RODRIGUES FORMULA
}

\author{
Halil GEZER and Cem KAANOĞLU
}

Cyprus International University, Faculty of Arts and Sciences, Department of Basic Sciences and Humanities, Nicosia, Mersin 10, TURKEY

\begin{abstract}
In this paper, we introduce and study a new family of analytic functions via Rodrigues formula. Some main properties, the generating function, various recurrence relations and differential properties of these functions are obtained. Furthermore, the differential equations are given for the subclasses of this family of analytic functions.
\end{abstract}

\section{INTRODUCTION}

Multiple orthogonal polynomials which are extensions of the orthogonal polynomials have been an important research area during the last few decades (see [6], 7], 8, 10, 11, 14]). These polynomials are closely related to Hermite-Padé approximation of a system of Markov functions [15. Also, the multiple orthogonal polynomials appear in random matrix theory, number theory, probability, approximation theory and mathematical physics (for examples see [1, [5], [9], [13, 15], [17]). These polynomials have some general properties such as Rodrigues formula and generating function. Very recently, the Rodrigues type generalizations of the multiple orthogonal polynomials have been studied in the papers [12] and [16].

There are many families of multiple orthogonal polynomials [8]. One of the classes of multiple orthogonal polynomials is the multiple Hermite polynomials which are the extension of the classical Hermite orthogonal polynomials.

For our purpose, we start by recalling the properties of the multiple Hermite polynomials. For the multi-index $\vec{n}=\left(n_{1}, n_{2}, \ldots, n_{r}\right) \in N^{r}$ and $\vec{\alpha}=\left(\alpha_{1}, \alpha_{2}, \ldots, \alpha_{r}\right)$ the multiple Hermite polynomials $\left\{H_{\vec{n}}^{(\delta, \vec{\alpha})}(x)\right\}$ of degree $|\vec{n}|=n_{1}+n_{2}+\ldots+n_{r}$

2020 Mathematics Subject Classification. Primary 33C05, 33C20; Secondary 33C45, 33C90.

Keywords and phrases. Multiple Hermite polynomials, Rodrigues formula, generating function, recurrence relation, differential equation.

\gezer@ciu.edu.tr-Corresponding author; kaanoglu@ciu.edu.tr

(D) 0000-0002-1248-989X; 0000-0001-7733-041X.

(C)2021 Ankara University Communications Faculty of Sciences University of Ankara-Series A1 Mathematics and Statistics 
were given in 14 by the Rodrigues formula

$$
H_{\vec{n}}^{(\delta, \vec{\alpha})}(x)=w_{r}^{-1}\left(w_{r} w_{r-1}^{-1}\left(\ldots\left(w_{2} w_{1}^{-1}\left(w_{1}\right)^{\left(n_{1}\right)}\right)^{\left(n_{2}\right)} \ldots\right)^{\left(n_{r-1}\right)}\right)^{\left(n_{r}\right)}
$$

where $w_{i}(x)=e^{\frac{\delta}{2} x^{2}+\alpha_{i} x}, \delta<0, \alpha_{i} \neq \alpha_{j}$ for $i \neq j$.

The orthogonality relation for the multiple Hermite polynomials $\left\{H_{\vec{n}}^{(\delta, \vec{\alpha})}(x)\right\}$ were given in 8$]$ as

$$
\int_{-\infty}^{\infty} H_{\vec{n}}^{(\delta, \vec{\alpha})}(x) x^{k} w_{i}(x) d x=0, k=0,1, \ldots, n_{i}-1 ; i=1,2, \ldots, r,
$$

with respect to $r$ weight functions $w_{i}(x)$.

In 12, the multiple Hermite polynomials were generalized via Rodrigues formula as

$$
G_{n_{1}, n_{2}}^{\left(\alpha, \alpha_{1}, \alpha_{2}\right)}(x ; k ; p)=x^{-\alpha} e^{p x^{k}} e^{-\alpha_{2} x} \frac{d^{n_{2}}}{d x^{n_{2}}} e^{\alpha_{2} x} e^{-\alpha_{1} x} \frac{d^{n_{1}}}{d x^{n_{1}}} x^{\alpha} e^{-p x^{k}} e^{\alpha_{1} x},
$$

where $p>0 ; \alpha, \alpha_{1}, \alpha_{2}>-1$ with $\alpha_{1} \neq \alpha_{2}$ and $k$ is a natural number.

The special case of (2) when $k=2, \alpha=0$ and $p=-\frac{\delta}{2}$ gives the Rodrigues formula of the multiple Hermite polynomials $H_{n_{1}, n_{2}}^{\left(\delta, \alpha_{1}, \alpha_{2}\right)}(x)$ given by (1).

Up to now, the generalizations of the multiple orthogonal polynomials via Rodrigues formula have been done by adding an extra parameter to the corresponding Rodrigues formula (see $[12,[16]$ ).

In this study, we introduce a new family of analytic functions via Rodrigues formula as

$$
H_{n_{1}, n_{2}, \ldots, n_{r}}(x)=B^{\Psi_{r+1}(x)-\Psi_{0}(x)} D^{n_{r}} B^{\Psi_{r}(x)} \ldots B^{\Psi_{3}(x)} D^{n_{2}} B^{\Psi_{2}(x)} D^{n_{1}} B^{\Psi_{0}(x)+\Psi_{1}(x)},
$$

where $\Psi_{i}(x), i=0,1, \ldots, r+1$ are analytic functions and $B>1$ is a constant. In particular case $r=2$, the Rodrigues formula (3) becomes

$$
H_{n_{1}, n_{2}}(x)=B^{\Psi_{3}(x)-\Psi_{0}(x)} D^{n_{2}} B^{\Psi_{2}(x)} D^{n_{1}} B^{\Psi_{0}(x)+\Psi_{1}(x)} .
$$

Note that, this type generalizations were done for some classical orthogonal polynomials by many authors (see [2], [3], 4]).

Remark 1. The particular case of Eq. (4) when $B=e, \Psi_{0}(x)=\frac{\delta}{2} x^{2}, \Psi_{1}(x)=$ $\alpha_{1} x, \Psi_{2}(x)=\left(\alpha_{2}-\alpha_{1}\right) x$ and $\Psi_{3}(x)=-\alpha_{2} x$ gives the multiple Hermite polynomials which was studied by D.W. Lee (see [14]], Eq. (6)). Also, in the case $B=e$, $\Psi_{0}(x)=-p x^{k}, \Psi_{1}(x)=\alpha_{1} x, \Psi_{2}(x)=\left(\alpha_{2}-\alpha_{1}\right) x$ and $\Psi_{3}(x)=-\alpha_{2} x$ the family of analytic functions given in (4) reduces to the special case $(\alpha=0)$ of the generalized multiple Hermite polynomial which is given in (2).

The paper is organized as follows: In section 2 we present the generating function, various recurrence relations and some differential properties for $H_{n_{1}, n_{2}}(x)$ which is given in Eq.(4). The differential equations for the particular cases of these functions are given in Section 3. 


\section{Generating Function and Recurrence Relations}

In this section we obtain generating function and various recurrence relations for the family defined in Eq.(4). The first result is given by the following theorem.

Theorem 2. Let $H_{n_{1}, n_{2}}(x)$ be the family of analytic functions defined by (4). Then the generating function is given by

$$
\begin{aligned}
G\left(x, t_{1}, t_{2}\right) & =\sum_{n_{1}, n_{2}=0}^{\infty} H_{n_{1}, n_{2}}(x) \frac{t_{1}^{n_{1}}}{n_{1} !} \frac{t_{2}^{n_{2}}}{n_{2} !} \\
& =B^{\Psi_{0}\left(x+t_{1}+t_{2}\right)-\Psi_{0}(x)+\Psi_{1}\left(x+t_{1}+t_{2}\right)+\Psi_{2}\left(x+t_{2}\right)+\Psi_{3}(x)},
\end{aligned}
$$

where $\Psi_{0}(x), \Psi_{1}(x), \Psi_{2}(x)$ and $\Psi_{3}(x)$ are analytic functions and $B>1$ is a constant.

Proof. By the Cauchy integral formula and the Rodrigues formula (4), we get

$$
\begin{aligned}
& \sum_{n_{1}, n_{2}=0}^{\infty} H_{n_{1}, n_{2}}(x) \frac{t_{1}^{n_{1}}}{n_{1} !} \frac{t_{2}^{n_{2}}}{n_{2} !} \\
= & \sum_{n_{2}=0}^{\infty} \sum_{n_{1}=0}^{\infty} B^{\Psi_{3}(x)-\Psi_{0}(x)} D^{n_{2}} B^{\Psi_{2}(x)} D^{n_{1}} B^{\Psi_{0}(x)+\Psi_{1}(x)} \frac{t_{1}^{n_{1}}}{n_{1} !} \frac{t_{2}^{n_{2}}}{n_{2} !} \\
= & \sum_{n_{2}=0}^{\infty} B^{\Psi_{3}(x)-\Psi_{0}(x)} D^{n_{2}} B^{\Psi_{2}(x)} \frac{t_{2}^{n_{2}}}{n_{2} !} \frac{1}{2 \pi i} \oint_{C_{1}} \frac{B^{\Psi_{0}(z)+\Psi_{1}(z)}}{z-x} \sum_{n_{1}=0}^{\infty}\left(\frac{t_{1}}{z-x}\right)^{n_{1}} d z \\
= & \sum_{n_{2}=0}^{\infty} B^{\Psi_{3}(x)-\Psi_{0}(x)} D^{n_{2}} B^{\Psi_{2}(x)} \frac{t_{2}^{n_{2}}}{n_{2} !} \frac{1}{2 \pi i} \oint_{C_{1}} \frac{B^{\Psi_{0}(z)+\Psi_{1}(z)}}{z-\left(x+t_{1}\right)} d z \\
= & \sum_{n_{2}=0}^{\infty} B^{\Psi_{3}(x)-\Psi_{0}(x)} D^{n_{2}} B^{\Psi_{2}(x)+\Psi_{0}\left(x+t_{1}\right)+\Psi_{1}\left(x+t_{1}\right)} \frac{t_{2}^{n_{2}}}{n_{2} !} \\
= & \left.B^{\Psi_{3}(x)-\Psi_{0}(x)} \frac{1}{2 \pi i} \oint_{C_{2}} \frac{B^{\Psi_{0}\left(\eta+t_{1}\right)+\Psi_{1}\left(\eta+t_{1}\right)+\Psi_{2}(\eta)}}{\eta-x} \sum_{n_{2}=0}^{\infty} \frac{t_{2}}{\eta-x}\right)^{n_{2}} d \eta \\
= & B^{\Psi_{0}\left(x+t_{1}+t_{2}\right)-\Psi_{0}(x)+\Psi_{1}\left(x+t_{1}+t_{2}\right)+\Psi_{2}\left(x+t_{2}\right)+\Psi_{3}(x)},
\end{aligned}
$$

where $C_{1}$ is a circle in the complex $z$-plane, cut along the negative real axis, centered at $z=x+t_{1}$ with radius $\epsilon>0$ (sufficiently small), which is described in the positive direction (counter-clockwise) and $C_{2}$ is a circle, centered at $\eta=x+t_{2}$ in the complex $\eta$-plane, cut along the negative real axis, with sufficiently small radius which is described in the positive direction (counter-clockwise) and $\left|\frac{t_{1}}{z-x}\right|<1,\left|\frac{t_{2}}{\eta-x}\right|<1$. Whence the result. 
Remark 3. Assume $B=e, \Psi_{0}(x)=\frac{\delta}{2} x^{2}, \Psi_{1}(x)=\alpha_{1} x, \Psi_{2}(x)=\left(\alpha_{2}-\alpha_{1}\right) x$ and $\Psi_{3}(x)=-\alpha_{2} x$ in Eq. (5) then we get the generating function for the multiple Hermite polynomials which was already found by D.W. Lee [14].

The main recurrence relations of this paper are given in the following theorem. As a consequence of these recurrence relations we are going to find the differential equation for the special cases of the class of analytic functions $H_{n_{1}, n_{2}}(x)$.

Theorem 4. For the family of functions $H_{n_{1}, n_{2}}(x)$ given in Eq. (4), we have the following recurrence relations

$$
\begin{aligned}
& H_{n_{1}+1, n_{2}}(x) \\
& =\ln B\left(\sum_{p=0}^{n_{1}} \sum_{p_{1}=0}^{n_{2}}\left(\begin{array}{c}
n_{1} \\
p
\end{array}\right)\left(\begin{array}{c}
n_{2} \\
p_{1}
\end{array}\right) H_{n_{1}-p, n_{2}-p_{1}}(x) \frac{\partial^{p+p_{1}+1}\left(\Psi_{0}(x)+\Psi_{1}(x)\right)}{\partial t_{1}^{p+1} \partial t_{2}^{p_{1}}}\right), \\
& H_{n_{1}, n_{2}+1}(x) \\
& =\ln B\left(\sum_{p=0}^{n_{1}} \sum_{p_{1}=0}^{n_{2}}\left(\begin{array}{c}
n_{1} \\
p
\end{array}\right)\left(\begin{array}{c}
n_{2} \\
p_{1}
\end{array}\right) H_{n_{1}-p, n_{2}-p_{1}}(x) \frac{\partial^{p+p_{1}+1}\left(\Psi_{0}(x)+\Psi_{1}(x)\right)}{\partial t_{1}^{p} \partial t_{2}^{p_{1}+1}}\right. \\
& \left.+\sum_{m=0}^{n_{2}}\left(\begin{array}{c}
n_{2} \\
m
\end{array}\right) H_{n_{1}, n_{2}-m}(x) \frac{\partial^{m+1} \Psi_{2}(x)}{\partial t_{2}^{m+1}}\right)
\end{aligned}
$$

and

$$
\begin{aligned}
& H_{n_{1}, n_{2}}^{\prime}(x) \\
= & \ln B\left(\sum_{p=0}^{n_{1}} \sum_{p_{1}=0}^{n_{2}}\left(\begin{array}{c}
n_{1} \\
p
\end{array}\right)\left(\begin{array}{c}
n_{2} \\
p_{1}
\end{array}\right) H_{n_{1}-p, n_{2}-p_{1}}(x) \frac{\partial^{p+p_{1}+1}\left(\Psi_{0}(x)+\Psi_{1}(x)\right)}{\partial t_{1}^{p+1} \partial t_{2}^{p_{1}}}\right. \\
+ & \sum_{m=0}^{n_{2}}\left(\begin{array}{c}
n_{2} \\
m
\end{array}\right) H_{n_{1}, n_{2}-m}(x) \frac{\partial^{(m+1)} \Psi_{2}(x)}{\partial t_{2}^{(m+1)}} \\
+ & \left.\left(\Psi_{3}^{\prime}(x)-\Psi_{0}^{\prime}(x)\right) H_{n_{1}, n_{2}}(x)\right) .
\end{aligned}
$$

Proof. Let $\Psi\left(x, t_{1}, t_{2}\right)$ be an analytic function. The Taylor series expansion of $\Psi\left(x, t_{1}, t_{2}\right)$ around $t_{1}, t_{2}=0$ is defined and represented as

$$
\Psi\left(x, t_{1}, t_{2}\right)=\left.\sum_{n=0}^{\infty} \frac{1}{n !} \sum_{k=0}^{n}\left(\begin{array}{l}
n \\
k
\end{array}\right) \frac{\partial^{n} \Psi\left(x, t_{1}, t_{2}\right)}{\partial t_{1}^{n-k} \partial t_{2}^{k}}\right|_{\left(t_{1}=0, t_{2}=0\right)} t_{1}^{n-k} t_{2}^{k} .
$$

For convenience let $\left.\frac{\partial^{n} \Psi\left(x, t_{1}, t_{2}\right)}{\partial t_{1}^{n-k} \partial t_{2}^{k}}\right|_{\left(t_{1}=0, t_{2}=0\right)}=\frac{\partial^{n} \Psi(x)}{\partial t_{1}^{n-k} \partial t_{2}^{k}}$.

The generating function $G\left(x, t_{1}, t_{2}\right)$ gives a way to derive the recurrence relations. 
By differentiating Eq. (5) with respect to $t_{1}$, one has

$$
\frac{\partial}{\partial t_{1}} G\left(x, t_{1}, t_{2}\right)=\ln B\left(\Psi_{0}^{\prime}\left(x+t_{1}+t_{2}\right)+\Psi_{1}^{\prime}\left(x+t_{1}+t_{2}\right)\right) G\left(x, t_{1}, t_{2}\right) .
$$

Now, if we substitute the Taylor series of the analytic functions $\Psi_{0}^{\prime}\left(x+t_{1}+t_{2}\right)$ and $\Psi_{1}^{\prime}\left(x+t_{1}+t_{2}\right)$ around $t_{1}, t_{2}=0$ into the above equation we get

$$
\begin{aligned}
& \sum_{n_{1}, n_{2}=0}^{\infty} H_{n_{1}+1, n_{2}}(x) \frac{t_{1}^{n_{1}}}{n_{1} !} \frac{t_{2}^{n_{2}}}{n_{2} !} \\
= & \ln B\left(\sum_{p=0}^{\infty} \frac{1}{p !} \sum_{p_{1}=0}^{p}\left(\begin{array}{c}
p \\
p_{1}
\end{array}\right) \frac{\partial^{p+1} \Psi_{0}(x)}{\partial t_{1}^{p-p_{1}+1} \partial t_{2}^{p_{2}}} t_{1}^{p-p_{1}} t_{2}^{p_{1}}\right. \\
+ & \left.\sum_{k=0}^{\infty} \frac{1}{k !} \sum_{k_{1}=0}^{k}\left(\begin{array}{c}
k \\
k_{1}
\end{array}\right) \frac{\partial^{k+1} \Psi_{1}(x)}{\partial t_{1}^{k-k_{1}+1} \partial t_{2}^{k_{1}}} t_{1}^{k-k_{1}} t_{2}^{k_{1}}\right) \sum_{n_{1}, n_{2}=0}^{\infty} H_{n_{1}, n_{2}}(x) \frac{t_{1}^{n_{1}}}{n_{1} !} \frac{t_{2}^{n_{2}}}{n_{2} !} \\
= & \ln B\left(\sum_{n_{1}, n_{2}=0}^{\infty} \sum_{p=0}^{\infty} \sum_{p_{1}=0}^{\infty} \frac{1}{\left(p+p_{1}\right) !}\left(\begin{array}{c}
p+p_{1} \\
p_{1}
\end{array}\right) H_{n_{1}, n_{2}}(x) \frac{\partial^{p+p_{1}+1} \Psi_{0}(x)}{\partial t_{1}^{p+1} \partial t_{2}^{p_{1}}} \frac{t_{1}^{n_{1}+p}}{n_{1} !} \frac{t_{2}^{n_{2}+p_{1}}}{n_{2} !}\right. \\
+ & \left.\sum_{n_{1}, n_{2}=0}^{\infty} \sum_{k=0}^{\infty} \sum_{k_{1}=0}^{\infty} \frac{1}{\left(k+k_{1}\right) !}\left(\begin{array}{c}
k+k_{1} \\
k_{1}
\end{array}\right) H_{n_{1}, n_{2}}(x) \frac{\partial^{k+k_{1}+1} \Psi_{1}(x)}{\partial t_{1}^{k+1} \partial t_{2}^{k_{1}}} \frac{t_{1}^{n_{1}+k}}{n_{1} !} \frac{t_{2}^{n_{2}+k_{1}}}{n_{2} !}\right) .
\end{aligned}
$$

If we set $n_{1} \rightarrow n_{1}-p$ and $n_{2} \rightarrow n_{2}-p_{1}$ for the first set of series and if we set $n_{1} \rightarrow n_{1}-k$ and $n_{2} \rightarrow n_{2}-k_{1}$ for the second set of series to the right of the last equation we get

$$
\begin{aligned}
& \sum_{n_{1}, n_{2}=0}^{\infty} H_{n_{1}+1, n_{2}}(x) \frac{t_{1}^{n_{1}}}{n_{1} !} \frac{t_{2}^{n_{2}}}{n_{2} !} \\
= & \ln B\left(\sum_{n_{1}, n_{2}=0}^{\infty} \sum_{p=0}^{n_{1}} \sum_{p_{1}=0}^{n_{2}} \frac{1}{\left(p+p_{1}\right) !}\left(\begin{array}{c}
p+p_{1} \\
p_{1}
\end{array}\right)\right. \\
\times \quad & H_{n_{1}-p, n_{2}-p_{1}}(x) \frac{\partial^{p+p_{1}+1} \Psi_{0}(x)}{\partial t_{1}^{p+1} \partial t_{2}^{p_{1}}} \frac{t_{1}^{n_{1}}}{\left(n_{1}-p\right) !} \frac{t_{2}^{n_{2}}}{\left(n_{2}-p_{1}\right) !} \\
+ & \sum_{n_{1}, n_{2}=0}^{\infty} \sum_{k=0}^{n_{1}} \sum_{k_{1}=0}^{n_{2}} \frac{1}{\left(k+k_{1}\right) !}\left(\begin{array}{c}
k+k_{1} \\
k_{1}
\end{array}\right) \\
\times \quad & \left.H_{n_{1}-k, n_{2}-k_{1}}(x) \frac{\partial^{k+k_{1}+1} \Psi_{1}(x)}{\partial t_{1}^{k+1} \partial t_{2}^{k_{1}}} \frac{t_{1}^{n_{1}}}{\left(n_{1}-k\right) !} \frac{t_{2}^{n_{2}}}{\left(n_{2}-k_{1}\right) !}\right) .
\end{aligned}
$$

If we compare the coefficients of $t_{1}^{n_{1}}$ and $t_{2}^{n_{2}}$ then we get

$$
\frac{1}{n_{1} ! n_{2} !} H_{n_{1}+1, n_{2}}(x)
$$




$$
\begin{aligned}
& =\ln B\left(\sum_{p=0}^{n_{1}} \sum_{p_{1}=0}^{n_{2}} \frac{1}{\left(p+p_{1}\right) !}\left(\begin{array}{c}
p+p_{1} \\
p_{1}
\end{array}\right)\right. \\
& \times \quad H_{n_{1}-p, n_{2}-p_{1}}(x) \frac{\partial^{p+p_{1}+1} \Psi_{0}(x)}{\partial t_{1}^{p+1} \partial t_{2}^{p_{1}}} \frac{1}{\left(n_{1}-p\right) !} \frac{1}{\left(n_{2}-p_{1}\right) !} \\
& +\sum_{k=0}^{n_{1}} \sum_{k_{1}=0}^{n_{2}} \frac{1}{\left(k+k_{1}\right) !}\left(\begin{array}{c}
k+k_{1} \\
k_{1}
\end{array}\right) \\
& \left.\times \quad H_{n_{1}-k, n_{2}-k_{1}}(x) \frac{\partial^{k+k_{1}+1} \Psi_{1}(x)}{\partial t_{1}^{k+1} \partial t_{2}^{k_{1}}} \frac{1}{\left(n_{1}-k\right) !} \frac{1}{\left(n_{2}-k_{1}\right) !}\right),
\end{aligned}
$$

which gives the proof of the first part. On the other hand, if we consider the partial derivatives, we obtain

$$
\frac{\partial}{\partial t_{2}} G\left(x, t_{1}, t_{2}\right)=\ln B\left(\Psi_{0}^{\prime}\left(x+t_{1}+t_{2}\right)+\Psi_{1}^{\prime}\left(x+t_{1}+t_{2}\right)+\Psi_{2}^{\prime}\left(x+t_{2}\right)\right) G\left(x, t_{1}, t_{2}\right)
$$

and

$$
\begin{aligned}
\frac{\partial}{\partial x} G\left(x, t_{1}, t_{2}\right) & =\ln B\left(\Psi_{0}^{\prime}\left(x+t_{1}+t_{2}\right)-\Psi_{0}^{\prime}(x)\right. \\
& \left.+\Psi_{1}^{\prime}\left(x+t_{1}+t_{2}\right)+\Psi_{2}^{\prime}\left(x+t_{2}\right)+\Psi_{3}^{\prime}(x)\right) G\left(x, t_{1}, t_{2}\right),
\end{aligned}
$$

respectively and use similar techniques as in the proof of first part then we prove second and third parts.

In the following corollary, we give more recurrence relations for $H_{n_{1}, n_{2}}(x)$.

Corollary 5. The following recurrence relations hold true for $H_{n_{1}, n_{2}}(x)$,

$$
\begin{aligned}
H_{n_{1}+1, n_{2}}(x) & =H_{n_{1}, n_{2}}^{\prime}(x)+\ln B\left(\Psi_{0}^{\prime}(x)-\Psi_{3}^{\prime}(x)\right) H_{n_{1}, n_{2}}(x) \\
& -\ln B\left(\sum_{l=0}^{n_{2}}\left(\begin{array}{c}
n_{2} \\
l
\end{array}\right) H_{n_{1}, n_{2}-l}(x) \Psi_{2}^{(l+1)}(x)\right), \\
H_{n_{1}, n_{2}+1}(x) & =H_{n_{1}, n_{2}}^{\prime}(x)+\ln B\left(\Psi_{0}^{\prime}(x)-\Psi_{3}^{\prime}(x)\right) H_{n_{1}, n_{2}}(x),
\end{aligned}
$$

and

$$
H_{n_{1}+1, n_{2}}(x)=H_{n_{1}, n_{2}+1}(x)-\ln B\left(\sum_{l=0}^{n_{2}}\left(\begin{array}{c}
n_{2} \\
l
\end{array}\right) H_{n_{1}, n_{2}-l}(x) \Psi_{2}^{(l+1)}(x)\right) .
$$

Proof. We have

$$
\frac{\partial}{\partial x} G\left(x, t_{1}, t_{2}\right)=\frac{\partial}{\partial t_{1}} G\left(x, t_{1}, t_{2}\right)+\ln B\left(\Psi_{2}^{\prime}\left(x+t_{2}\right)-\Psi_{0}^{\prime}(x)+\Psi_{3}^{\prime}(x)\right) G\left(x, t_{1}, t_{2}\right),
$$


if we consider the Taylor series of $\Psi_{2}^{\prime}\left(x+t_{2}\right)$ around $t_{2}=0$ then we get

$$
\begin{aligned}
& \sum_{n_{1}, n_{2}=0}^{\infty} H_{n_{1}, n_{2}}^{\prime}(x) \frac{t_{1}^{n_{1}}}{n_{1} !} \frac{t_{2}^{n_{2}}}{n_{2} !} \\
= & \sum_{n_{1}, n_{2}=0}^{\infty} H_{n_{1}+1, n_{2}}(x) \frac{t_{1}^{n_{1}}}{n_{1} !} \frac{t_{2}^{n_{2}}}{n_{2} !}+\ln B\left(\sum_{n_{1}, n_{2}=0}^{\infty} \sum_{l=0}^{\infty} H_{n_{1}, n_{2}}(x) \Psi_{2}^{(l+1)}(x) \frac{t_{1}^{n_{1}}}{n_{1} !} \frac{t_{2}^{n_{2}+l}}{n_{2} ! l !}\right) \\
+ & \ln B\left(\Psi_{3}^{\prime}(x)-\Psi_{0}^{\prime}(x)\right) \sum_{n_{1}, n_{2}=0}^{\infty} H_{n_{1}, n_{2}}(x) \frac{t_{1}^{n_{1}}}{n_{1} !} \frac{t_{2}^{n_{2}}}{n_{2} !} .
\end{aligned}
$$

If we replace $n_{2}$ with $n_{2}-l$ into the second set of sums on the right and compare the coefficients of $t_{1}^{n_{1}} t_{2}^{n_{2}}$ we have

$$
\begin{aligned}
\frac{1}{n_{1} !} \frac{1}{n_{2} !} H_{n_{1}+1, n_{2}}(x)= & \frac{1}{n_{1} !} \frac{1}{n_{2} !} H_{n_{1}, n_{2}}^{\prime}(x) \\
& +\frac{1}{n_{1} !} \frac{1}{n_{2} !} \ln B\left(\Psi_{0}^{\prime}(x)-\Psi_{3}^{\prime}(x)\right) H_{n_{1}, n_{2}}(x) \\
& -\ln B\left(\sum_{l=0}^{n_{2}} H_{n_{1}, n_{2}-l}(x) \Psi_{2}^{(l+1)}(x) \frac{1}{n_{1} !} \frac{1}{\left(n_{2}-l\right) !} \frac{1}{l !}\right),
\end{aligned}
$$

which proves the first equality. Furthermore, if we consider the relations

$$
\frac{\partial}{\partial x} G\left(x, t_{1}, t_{2}\right)=\frac{\partial}{\partial t_{2}} G\left(x, t_{1}, t_{2}\right)+\ln B\left(\Psi_{3}^{\prime}(x)-\Psi_{0}^{\prime}(x)\right) G\left(x, t_{1}, t_{2}\right)
$$

and

$$
\frac{\partial}{\partial t_{1}} G\left(x, t_{1}, t_{2}\right)=\frac{\partial}{\partial t_{2}} G\left(x, t_{1}, t_{2}\right)+\ln B \Psi_{2}^{\prime}\left(x+t_{2}\right) G\left(x, t_{1}, t_{2}\right),
$$

respectively then similar calculations help us to prove the another two results. Note that the another way to prove this corollary is to compare the recurrence relations which are given in Theorem 4.

\section{Some particular Results and Differential Equation}

It should be noted that, if the functions $\Psi_{0}(x), \Psi_{1}(x), \Psi_{2}(x)$ and $\Psi_{3}(x)$ are selected by means of analytic functions then a set of recurrence relations and differential properties can be given for the corresponding $H_{n_{1}, n_{2}}(x)$. As a consequence of these results the differential equation for $H_{n_{1}, n_{2}}(x)$ can be obtained.

The first set of particular results is given in the following corollary.

Corollary 6. Assume that $\Psi_{0}(x)$ is a polynomial of degree 2 and $\Psi_{1}(x), \Psi_{2}(x)$, $\Psi_{3}(x)$ are polynomials of degree 1 . Then the family of analytic functions $H_{n_{1}, n_{2}}(x)$ satisfies the folllowing recurence relations:

$$
H_{n_{1}+1, n_{2}}(x)=H_{n_{1}, n_{2}}^{\prime}(x)+\ln B\left(\Psi_{0}^{\prime}(x)-\Psi_{3}^{\prime}(x)-\Psi_{2}^{\prime}(x)\right) H_{n_{1}, n_{2}}(x),
$$




$$
\begin{aligned}
& H_{n_{1}, n_{2}+1}(x)=H_{n_{1}, n_{2}}^{\prime}(x)+\ln B\left(\Psi_{0}^{\prime}(x)-\Psi_{3}^{\prime}(x)\right) H_{n_{1}, n_{2}}(x), \\
& H_{n_{1}+1, n_{2}}^{\prime}(x)=H_{n_{1}, n_{2}}^{\prime \prime}(x)+\ln B\left(\Psi_{0}^{\prime \prime}(x)\right) H_{n_{1}, n_{2}}(x) \\
& +\ln B\left(\Psi_{0}^{\prime}(x)-\Psi_{3}^{\prime}(x)-\Psi_{2}^{\prime}(x)\right) H_{n_{1}, n_{2}}^{\prime}(x), \\
& H_{n_{1}, n_{2}+1}^{\prime}(x)=H_{n_{1}, n_{2}}^{\prime \prime}(x)+\ln B\left(\Psi_{0}^{\prime \prime}(x)\right) H_{n_{1}, n_{2}}(x) \\
& +\ln B\left(\Psi_{0}^{\prime}(x)-\Psi_{3}^{\prime}(x)\right) H_{n_{1}, n_{2}}^{\prime}(x), \\
& H_{n_{1}+1, n_{2}}^{\prime \prime}(x)=H_{n_{1}, n_{2}}^{\prime \prime \prime}(x)+2 \ln B\left(\Psi_{0}^{\prime \prime}(x)\right) H_{n_{1}, n_{2}}^{\prime}(x) \\
& +\ln B\left(\Psi_{0}^{\prime}(x)-\Psi_{3}^{\prime}(x)-\Psi_{2}^{\prime}(x)\right) H_{n_{1}, n_{2}}^{\prime \prime}(x), \\
& H_{n_{1}, n_{2}+1}^{\prime \prime}(x)=H_{n_{1}, n_{2}}^{\prime \prime \prime}(x)+2 \ln B\left(\Psi_{0}^{\prime \prime}(x)\right) H_{n_{1}, n_{2}}^{\prime}(x) \\
& +\ln B\left(\Psi_{0}^{\prime}(x)-\Psi_{3}^{\prime}(x)\right) H_{n_{1}, n_{2}}^{\prime \prime}(x), \\
& H_{n_{1}+1, n_{2}+1}(x)=H_{n_{1}, n_{2}}^{\prime \prime}(x)+\ln B\left(2 \Psi_{0}^{\prime}(x)-2 \Psi_{3}^{\prime}-\Psi_{2}^{\prime}(x)\right) H_{n_{1}, n_{2}}^{\prime}(x) \\
& +\ln B\left(\Psi_{0}^{\prime \prime}(x)+\ln B\left(\Psi_{0}^{\prime}(x)-\Psi_{3}^{\prime}(x)\right.\right. \\
& \left.\left.-\Psi_{2}^{\prime}(x)\right)\left(\Psi_{0}^{\prime}(x)-\Psi_{3}^{\prime}(x)\right)\right) H_{n_{1}, n_{2}}(x) \\
& H_{n_{1}+1, n_{2}+1}^{\prime}(x)=H_{n_{1}, n_{2}}^{\prime \prime \prime}(x)+\ln B\left(2 \Psi_{0}^{\prime}(x)-2 \Psi_{3}^{\prime}(x)-\Psi_{2}^{\prime}(x)\right) H_{n_{1}, n_{2}}^{\prime \prime}(x) \\
& +\ln B\left(3 \Psi_{0}^{\prime \prime}(x)+\ln B\left(\Psi_{0}^{\prime}(x)-\Psi_{3}^{\prime}(x)\right.\right. \\
& \left.\left.-\Psi_{2}^{\prime}(x)\right)\left(\Psi_{0}^{\prime}(x)-\Psi_{3}^{\prime}(x)\right)\right) H_{n_{1}, n_{2}}^{\prime}(x) \\
& +\ln B\left(\ln B\left(\Psi_{0}^{\prime \prime}(x)\right)\left(\Psi_{0}^{\prime}(x)-\Psi_{3}^{\prime}(x)\right)\right. \\
& \left.+\ln B\left(\Psi_{0}^{\prime}(x)-\Psi_{3}^{\prime}(x)-\Psi_{2}^{\prime}(x)\right)\left(\Psi_{0}^{\prime \prime}(x)\right)\right) H_{n_{1}, n_{2}}(x) .
\end{aligned}
$$

Proof. The results can be easily seen by substituting $\Psi_{0}(x), \Psi_{1}(x), \Psi_{2}(x)$ and $\Psi_{3}(x)$ into the relations which are given in Theorem 4 and Corollary 5. 
Note that, under the assumptions given in Remark 1, we can easily write the above recurrence relations for the multiple Hermite polynomials which some of them were also calculated in [14].

In the following theorem, we give a differential equation for the special case of $H_{n_{1}, n_{2}}(x)$ which is defined in Eq. (4).

Theorem 7. Let $\Psi_{0}(x)$ be a polynomial of degree 2 and let $\Psi_{1}(x), \Psi_{2}(x), \Psi_{3}(x)$ be polynomials of degree 1 . Then the family of functions $H_{n_{1}, n_{2}}(x)$ satisfies the following differential equation

$$
\begin{aligned}
& H_{n_{1}, n_{2}}^{\prime \prime \prime}(x)+\ln B\left\{2 \Psi_{0}^{\prime}(x)-3 \Psi_{3}^{\prime}(x)-2 \Psi_{2}^{\prime}(x)-\Psi_{1}^{\prime}(x)\right\} H_{n_{1}, n_{2}}^{\prime \prime}(x) \\
& +\ln B\left\{3 \Psi_{0}^{\prime \prime}(x)+\ln B\left(\Psi_{0}^{\prime}(x)-\Psi_{3}^{\prime}(x)-\Psi_{2}^{\prime}(x)\right)\left(\Psi_{0}^{\prime}(x)-\Psi_{3}^{\prime}(x)\right)\right. \\
& -\ln B\left(\Psi_{1}^{\prime}(x)+\Psi_{2}^{\prime}(x)+\Psi_{3}^{\prime}(x)\right)\left(2 \Psi_{0}^{\prime}(x)-2 \Psi_{3}^{\prime}(x)-\Psi_{2}^{\prime}(x)\right) \\
& \left.-\left(n_{2}+1\right) \Psi_{0}^{\prime \prime}(x)-\left(n_{1}+1\right) \Psi_{0}^{\prime \prime}(x)\right\} H_{n_{1}, n_{2}}^{\prime}(x) \\
& +\ln B\left\{\ln B \Psi_{0}^{\prime \prime}(x)\left(\Psi_{0}^{\prime}(x)-\Psi_{3}^{\prime}(x)\right)+\ln B\left(\Psi_{0}^{\prime}(x)-\Psi_{3}^{\prime}(x)-\Psi_{2}^{\prime}(x)\right) \Psi_{0}^{\prime \prime}(x)\right. \\
& -\ln B\left(\Psi_{1}^{\prime}(x)+\Psi_{2}^{\prime}(x)+\Psi_{3}^{\prime}(x)\right) \\
& \times\left(\Psi_{0}^{\prime \prime}(x)+\ln B\left(\Psi_{0}^{\prime}(x)-\Psi_{3}^{\prime}(x)-\Psi_{2}^{\prime}(x)\right)\left(\Psi_{0}^{\prime}(x)-\Psi_{3}^{\prime}(x)\right)\right) \\
& -\ln B\left(n_{2}+1\right) \Psi_{0}^{\prime \prime}(x)\left(\Psi_{0}^{\prime}(x)-\Psi_{3}^{\prime}(x)-\Psi_{2}^{\prime}(x)\right) \\
& \left.-\ln B\left(n_{1}+1\right) \Psi_{0}^{\prime \prime}(x)\left(\Psi_{0}^{\prime}(x)-\Psi_{3}^{\prime}(x)\right)\right\} H_{n_{1}, n_{2}}(x) \\
& =0 .
\end{aligned}
$$

Proof. Consider the third relation in Theorem 4 then

$$
\begin{aligned}
\frac{1}{n_{1} ! n_{2} !} H_{n_{1}, n_{2}}^{\prime}(x)= & \ln B\left(\left(\Psi_{1}^{\prime}(x)+\Psi_{2}^{\prime}(x)+\Psi_{3}^{\prime}(x)\right) H_{n_{1}, n_{2}}(x) \frac{1}{n_{1} !} \frac{1}{n_{2} !}\right. \\
& +H_{n_{1}, n_{2}-1}(x) \Psi_{0}^{\prime \prime}(x) \frac{1}{n_{1} !} \frac{1}{\left(n_{2}-1\right) !} \\
& \left.+H_{n_{1}-1, n_{2}}(x) \Psi_{0}^{\prime \prime}(x) \frac{1}{\left(n_{1}-1\right) !} \frac{1}{n_{2} !}\right) .
\end{aligned}
$$

Setting $n_{1} \rightarrow n_{1}+1$ and $n_{2} \rightarrow n_{2}+1$ then

$$
H_{n_{1}+1, n_{2}+1}^{\prime}(x)=\ln B\left(\left(\Psi_{1}^{\prime}(x)+\Psi_{2}^{\prime}(x)+\Psi_{3}^{\prime}(x)\right) H_{n_{1}+1, n_{2}+1}(x)\right.
$$




$$
\left.+\left(n_{2}+1\right) H_{n_{1}+1, n_{2}}(x) \Psi_{0}^{\prime \prime}(x)+\left(n_{1}+1\right) H_{n_{1}, n_{2}+1}(x) \Psi_{0}^{\prime \prime}(x)\right) .
$$

The results which were found in Corollary 6 give,

$$
\begin{aligned}
& H_{n_{1}, n_{2}}^{\prime \prime \prime}(x)+\ln B\left(2 \Psi_{0}^{\prime}(x)-2 \Psi_{3}^{\prime}(x)-\Psi_{2}^{\prime}(x)\right) H_{n_{1}, n_{2}}^{\prime \prime}(x) \\
+ & \ln B\left(3 \Psi_{0}^{\prime \prime}(x)+\ln B\left(\Psi_{0}^{\prime}(x)-\Psi_{3}^{\prime}(x)-\Psi_{2}^{\prime}(x)\right)\left(\Psi_{0}^{\prime}(x)-\Psi_{3}^{\prime}(x)\right)\right) H_{n_{1}, n_{2}}^{\prime}(x) \\
+ & \ln B\left(\ln B\left(\Psi_{0}^{\prime \prime}(x)\right)\left(\Psi_{0}^{\prime}(x)-\Psi_{3}^{\prime}(x)\right)+\ln B\left(\Psi_{0}^{\prime}(x)-\Psi_{3}^{\prime}(x)-\Psi_{2}^{\prime}(x)\right)\left(\Psi_{0}^{\prime \prime}(x)\right)\right) H_{n_{1}, n_{2}}(x) \\
= & \ln B\left\{( \Psi _ { 1 } ^ { \prime } ( x ) + \Psi _ { 2 } ^ { \prime } ( x ) + \Psi _ { 3 } ^ { \prime } ( x ) ) \left[H_{n_{1}, n_{2}}^{\prime \prime}(x)+\ln B\left(2 \Psi_{0}^{\prime}(x)-2 \Psi_{3}^{\prime}(x)-\Psi_{2}^{\prime}(x)\right) H_{n_{1}, n_{2}}^{\prime}(x)\right.\right. \\
+ & \left.\ln B\left(\Psi_{0}^{\prime \prime}(x)+\ln B\left(\Psi_{0}^{\prime}(x)-\Psi_{3}^{\prime}(x)-\Psi_{2}^{\prime}(x)\right)\left(\Psi_{0}^{\prime}(x)-\Psi_{3}^{\prime}(x)\right)\right) H_{n_{1}, n_{2}}(x)\right] \\
+ & \left(n_{2}+1\right) \Psi_{0}^{\prime \prime}(x)\left[H_{n_{1}, n_{2}}^{\prime}(x)+\ln B\left(\Psi_{0}^{\prime}(x)-\Psi_{3}^{\prime}(x)-\Psi_{2}^{\prime}(x)\right) H_{n_{1}, n_{2}}(x)\right] \\
+ & \left.\left(n_{1}+1\right) \Psi_{0}^{\prime \prime}(x)\left[H_{n_{1}, n_{2}}^{\prime}(x)+\ln B\left(\Psi_{0}^{\prime}(x)-\Psi_{3}^{\prime}(x)\right) H_{n_{1}, n_{2}}(x)\right]\right\} .
\end{aligned}
$$

Then the simple calculations yield that

$$
\begin{aligned}
& H_{n_{1}, n_{2}}^{\prime \prime \prime}(x)+\ln B\left\{2 \Psi_{0}^{\prime}(x)-3 \Psi_{3}^{\prime}(x)-2 \Psi_{2}^{\prime}(x)-\Psi_{1}^{\prime}(x)\right\} H_{n_{1}, n_{2}}^{\prime \prime}(x) \\
+ & \ln B\left\{3 \Psi_{0}^{\prime \prime}(x)+\ln B\left(\Psi_{0}^{\prime}(x)-\Psi_{3}^{\prime}(x)-\Psi_{2}^{\prime}(x)\right)\left(\Psi_{0}^{\prime}(x)-\Psi_{3}^{\prime}(x)\right)\right. \\
- & \ln B\left(\Psi_{1}^{\prime}(x)+\Psi_{2}^{\prime}(x)+\Psi_{3}^{\prime}(x)\right)\left(2 \Psi_{0}^{\prime}(x)-2 \Psi_{3}^{\prime}(x)-\Psi_{2}^{\prime}(x)\right) \\
- & \left.\left(n_{2}+1\right) \Psi_{0}^{\prime \prime}(x)-\left(n_{1}+1\right) \Psi_{0}^{\prime \prime}(x)\right\} H_{n_{1}, n_{2}}^{\prime}(x) \\
+ & \ln B\left\{\ln B \Psi_{0}^{\prime \prime}(x)\left(\Psi_{0}^{\prime}(x)-\Psi_{3}^{\prime}(x)\right)+\ln B\left(\Psi_{0}^{\prime}(x)-\Psi_{3}^{\prime}(x)-\Psi_{2}^{\prime}(x)\right) \Psi_{0}^{\prime \prime}(x)\right. \\
- & \ln B\left(\Psi_{1}^{\prime}(x)+\Psi_{2}^{\prime}(x)+\Psi_{3}^{\prime}(x)\right) \\
\times & \left(\Psi_{0}^{\prime \prime}(x)+\ln B\left(\Psi_{0}^{\prime}(x)-\Psi_{3}^{\prime}(x)-\Psi_{2}^{\prime}(x)\right)\left(\Psi_{0}^{\prime}(x)-\Psi_{3}^{\prime}(x)\right)\right) \\
- & \ln B\left(n_{2}+1\right) \Psi_{0}^{\prime \prime}(x)\left(\Psi_{0}^{\prime}(x)-\Psi_{3}^{\prime}(x)-\Psi_{2}^{\prime}(x)\right) \\
- & \left.\ln B\left(n_{1}+1\right) \Psi_{0}^{\prime \prime}(x)\left(\Psi_{0}^{\prime}(x)-\Psi_{3}^{\prime}(x)\right)\right\} H_{n_{1}, n_{2}}(x)
\end{aligned}
$$


$=0$.

Hence the result.

Corollary 8. Let $\Psi_{0}(x)=a_{0} x^{2}+b_{0} x+c_{0}, \Psi_{1}(x)=b_{1} x+c_{1}, \Psi_{2}(x)=b_{2} x+c_{2}$ and $\Psi_{3}=b_{3} x+c_{3},\left(a_{0}, b_{0}, b_{1}, b_{2}, b_{3}, c_{0}, c_{1}, c_{2}\right.$ and $c_{3}$ are constants) then the family of functions defined by the Rodrigues formula

$$
H_{n_{1}, n_{2}}(x)=B^{b_{3} x+c_{3}-\left(a_{0} x^{2}+b_{0} x+c_{0}\right)} D^{n_{2}} B^{b_{2} x+c_{2}} D^{n_{1}} B^{a_{0} x^{2}+b_{0} x+c_{0}+b_{1} x+c_{1}}
$$

satisfies the following differential equation

$$
\begin{aligned}
& H_{n_{1}, n_{2}}^{\prime \prime \prime}(x)+\ln B\left\{2\left(2 a_{0} x+b_{0}\right)-3 b_{3}-2 b_{2}-b_{1}\right\} H_{n_{1}, n_{2}}^{\prime \prime}(x) \\
+ & \ln B\left\{6 a_{0}+\ln B\left(2 a_{0} x+b_{0}-b_{3}-b_{2}\right)\left(2 a_{0} x+b_{0}-b_{3}\right)\right. \\
- & \ln B\left(b_{1}+b_{2}+b_{3}\right)\left(2\left(2 a_{0} x+b_{0}\right)-2 b_{3}-b_{2}\right) \\
- & \left.\left(n_{2}+1\right) 2 a_{0}-\left(n_{1}+1\right) 2 a_{0}\right\} H_{n_{1}, n_{2}}^{\prime}(x) \\
+ & \ln B\left\{\ln B\left(2 a_{0}\right)\left(2 a_{0} x+b_{0}-b_{3}\right)+\ln B\left(2 a_{0} x+b_{0}-b_{3}-b_{2}\right)\left(2 a_{0}\right)\right. \\
- & \ln B\left(b_{1}+b_{2}+b_{3}\right)\left(2 a_{0}\right. \\
& \left.+\ln B\left(2 a_{0} x+b_{0}-b_{3}-b_{2}\right)\left(2 a_{0} x+b_{0}-b_{3}\right)\right) \\
- & \ln B\left(n_{2}+1\right) 2 a_{0}\left(2 a_{0} x+b_{0}-b_{3}-b_{2}\right) \\
- & \left.\ln B\left(n_{1}+1\right) 2 a_{0}\left(2 a_{0} x+b_{0}-b_{3}\right)\right\} H_{n_{1}, n_{2}}(x) \\
= & 0
\end{aligned}
$$

In the special case, it is obvious that the differential equation in Theorem 7 reduces to the differential equation of the multiple Hermite polynomials, which was also mentioned in 6 .

Corollary 9. (see [6] ) The differential equation for the multiple Hermite polynomials $y=H_{n_{1}, n_{2}}^{\left(\delta, \alpha_{1}, \alpha_{2}\right)}(x)$ is given by

$$
\begin{aligned}
& y^{\prime \prime \prime}(x)+\left\{2 \delta x+\alpha_{2}+\alpha_{1}\right\} y^{\prime \prime}(x)+\{3 \delta \\
& \left.\left.+\left(\delta x+\alpha_{1}\right)\right)\left(\delta x+\alpha_{2}\right)-\left(n_{2}+1\right) \delta-\left(n_{1}+1\right) \delta\right\} y^{\prime}(x)
\end{aligned}
$$




$$
\begin{aligned}
& +\left\{\delta\left(\delta x+\alpha_{2}\right)+\left(\delta x+\alpha_{1}\right) \delta-\left(n_{2}+1\right) \delta\left(\delta x+\alpha_{1}\right)\right. \\
& \left.-\quad\left(n_{1}+1\right) \delta\left(\delta x+\alpha_{2}\right)\right\} y(x) \\
& =0 .
\end{aligned}
$$

Note that, for every suitable choices of the analytic functions $\Psi_{0}(x), \Psi_{1}(x)$, $\Psi_{2}(x)$ and $\Psi_{3}(x)$, the relations in Corollary 6 and Theorem 7 can be recalculated and then these relations can be applied in order to give a different order differential equation for the corresponding family of analytic functions.

\section{Concluding Remarks}

In this paper, the family of analytic functions $H_{n_{1}, n_{2}}(x)$ via Rodrigues formula has been introduced and studied. In the particular case, the analytic function $H_{n_{1}, n_{2}}(x)$ is a Rodrigues formula type extension of the known multiple Hermite polynomials. We obtained various general recurrence relations and differential properties for these functions. In the particular case, we showed that our results are consistent with the known results which were found before for the multiple Hermite polynomials. Furthermore, in Section 3 we observe that if $\Psi_{0}(x), \Psi_{1}(x), \Psi_{2}(x)$ and $\Psi_{3}(x)$ are selected by means of analytic functions then we have different class of analytic functions defined by Rodrigues formula and it is possible to find different order linear homogeneous differential equation for the corresponding class of analytic functions. In this study, we have considered $r=2$ case of the class of polynomials given in Eq. (3). For different $r$ values, it is possible to use similar techniques to obtain the calculated relations. But, it is complicated to find this type long formulas.

Authors Contribution Statement All authors jointly worked on the results and they read and approved the final manuscript.

Declaration of Competing Interests The authors declare that they have no competing interest.

\section{REFERENCES}

[1] Adler, M., Van Moerbeke, P., Vanhaecke P., Moment matrices and multi-component KP, with applications to random matrix theory, Comm. Math. Phys, 286 (2009), 1-38.

[2] Aktaş, R., Altın, A., Taşdelen, F., A new family of analytic functions defined by means of Rodrigues type formula, Math. Slovaca, 68(3) (2018), 607-616.

[3] Aktaş, R., Altın, A., A generating function and some recurrence relations for a family of polynomials, Proceedings of the 12th WSEAS International conference on Applied Mathematics, (2007), 118-121.

[4] Altın, A., Aktaş, R., A class of polynomials in two variables, Mathematica Morovica, 14 (1) (2014), 1-14.

[5] Aptekarev, A. I., Strong asymptotics of multiple orthogonal polynomials for Nikishin systems, Math. Sb. 190(5) (1999), 3-44 (Russian); Sbornik Math. 190(5) (1999), 631-669. 
[6] Aptekarev, A.I., Branquinho, A., Van Assche, W., Multiple orthogonal polynomials for classical weights, Transectional American Mathematical Society, 355(10) (2003), 3887-3914.

[7] Van Assche, W., Yakubovich, S.B., Multiple orthogonal polynomials associated with Mcdonald functions, Integral Transforms Spec. Funct., 9,3(2000), 229-244.

[8] Van Assche, W., Coussement, Eds., Some classical multiple orthogonal polynomials, J. Comput. Appl. Math., 127 (2001), 317-347.

[9] Bleher, P.M., Kuijlaars, A.B.J., Random matrices with external source and multiple orthogonal polynomials, Internat. Math. Research Notices, 3 (2004), 109-129.

[10] Coussement, E., Van Assche, W., Multiple orthogonal polynomials associated with the modified Bessel functions of the first kind, Constructive Approximation, 19 (2003), 237-263.

[11] Coussement, E. and Van Assche, W., Some properties of multiple orthogonal polynomials associated with Macdonald functions, J. Comput. Appl. Math., 133(2001), 253-261.

[12] Kaanoğlu, C., Özarslan, M.A., Some properties of generalized multiple Hermite polynomials, J. Comput. Appl. Math., 235 (2011), 4878-4887.

[13] Kuijlaars, A.B.J., Van Assche, W., Wielonsky, F., Quadratic Hermite-Pade approximation to the exponential function: a Riemann-Hilbert approach, Constr. Approx. 21 (2005), 313-320.

[14] Lee, D.W., Properties of multiple Hermite and multiple Laguerre polynomials by the generating function, Integral Transforms Spec. Funct. 18 (2007), 855-869.

[15] Nikishin, E.M., Sorokin, V.V., Rational approximations and orthogonality, Translations of Mathematical Monographs (Providence, RI: American Mathematical Society), (1991), R192.

[16] Özarslan, M.A., Kaanoğlu, C., Some generalization of multiple Laguerre polynomials via Rodrigues formula, Ars Combinatoria, 123 (2015), 195-206.

[17] Varma, S., On a generalization of Szasz operators by multiple Appell polynomials, Stud. Univ. Babeş-Bolyai Math. 58(3) (2013), 361-369. 